\title{
STUDY OF SERUM NEOPTERIN, INTERLEUKIN-10 AND NEUTROPHIL TO LYMPHOCYTE RATIO IN CONTRAST INDUCED NEPHROPATHY
}

\author{
Mohamed Aly Fahmy Zanaty MD*, Yousef Ahmed Kadry MD*, Hisham Mohamed Omar MD** and \\ Mohamed Gomaa Abd el-Rehim MSc*. \\ "Nephrology Unit, Internal Medicine Department, Faculty of Medicine, Zagazig University, Egypt. \\ ${ }^{* *}$ Clinical Pathology Department, Faculty of Medicine, Zagazig University, Egypt.
}

\begin{abstract}
- Background: Recent improvements in radio-diagnostic procedures and in cardiovascular percutaneous interventions, together with increased life expectancy, have resulted in the subjection of an increasing number of patients to contrast medium-enhanced examinations or cardiac and angiographic procedure requiring iodinated contrast medium injection.

- Subjects and methods: This study was carried out at Internal Medicine, Cardiology, Radiology and Clinical Pathology departments, Zagazig University Hospitals. The study was approved by Institutional Review Board (IRB) and included a total of 60 patients.

- $\quad$ Based on the development of contrast induced nephropathy after IV administration of high osmolar contrast agent, Patients were classified into CIN and NO CIN groups. We measured serum neopterin and IL-10 and calculating neutrophil to lymphocyte ratio before contrast administration, 24 and 48 hours after in 45 patients underwent coronary angiography for diagnostic and therapeutic purposes and 15 patients underwent renal angiography.

- Results: About $25 \%$ of the present studied patients (14 patients) fulfilled the criteria of contrast nephropathy with more cases in the percutaneous coronary angiography group (11 patients).This study revealed that serum neopterin, IL 10 and neutrophil to lymphocyte ratio were higher in patients with CIN following IV contrast media at $24 \& 48$ hours compared to patients without CIN. In our study; we found increased preprocedural and post-procedural CRP, ESR and uric acid levels at 48 hours in patients with CIN. We found a decreased basal serum total bilirubin level in patients with CIN following IV contrast media compared to patients without CIN.

- Conclusion: Serum neopterin, IL 10 and neutrophil to lymphocyte ratio proved that they can be used as early biomarkers of contrast induced nephropathy instead of serum creatinine as they rise 24 hours before any change in the serum creatinine. Decreased serum total bilirubin levels had a higher incidence of CIN after the use of contrast media. Measuring ESR \& CRP levels at admission may offer additional assistance in predicting the development of CIN. Elevated serum uric acid level is independently associated with an increased risk of CIN.
\end{abstract}

- $\quad$ Abbreviations: $\mathbf{C I N}=$ Contrast induced nephropathy, $\mathbf{A K I}=$ Acute kidney injury, $\mathbf{C R P}=\mathrm{C}$-reactive protein, $\mathbf{N L R}=$ Neutrophil to lymphocyte ratio, $\mathbf{E S R}=$ Erythrocyte sedimentation rate, $\mathbf{U A}=$ Uric acid, $\mathbf{e G F R}=$ estimated glomerular filtration rate.

- Keywords: Contrast induced nephropathy; Neopterin; IL-10; Neutrophil to lymphocyte ratio.

- Corresponding author: Mohamed Gomaa Abd el-Rehim Mobile: 01064277675 Email: dr.mohamedgomaa500@yahoo.com.

\section{INTRODUCTION}

A cute kidney injury (AKI) represents a significant and devastating problem in clinical medicine. The incidence of AKI varies from $5 \%$ of hospitalized patients to $30-50 \%$ of patients in intensive care units (1).

With the increasing use of radio-contrast media in diagnostic and interventional procedures over the last 30 years, Contrast- induced nephropathy (CIN) has become the third leading cause of hospital-acquired acute renal failure (2).

CIN is usually defined as impairment of renal function occurring within $48 \mathrm{~h}$ after administration of contrast media and manifested by an absolute increase in serum creatinine concentration of at least $0.5 \mathrm{mg} / \mathrm{dl}$ or 
by a relative increase of at least $25 \%$ from the baseline value (3).

Two principal mechanisms have been reported for CIN pathogenesis: renal vasoconstriction resulting in medullary hypoxia and direct cytotoxic effects of the contrast agents. Following the exposure to the contrast agent, effects of various mediators (vasopressin and endothelin) and reduction in the production of nitric oxide lead to renal vasoconstriction, impaired vasodilation and reduced medullary blood flow (4).

Furthermore, tubular injury associated with oxygen radicals, pro-inflammatory cytokines and complement activity are another pathophysiologic events that result in tubular obstruction in the form of protein precipitates (5).

Experimental studies have shown a significant relationship between inflammation and AKI. First, renal vascular endothelium and tubular epithelium undergo structural and functional changes due to ischemia, sepsis or nephrotoxic effects. At the next stage, inflammatory cells such as macrophages, natural killer cells, lymphocytes and particularly neutrophils infiltrate damaged tissue leading to further renal destruction Active neutrophils trigger endothelial cellular damage that plays a role in reduced vasodilator response, elevated vasoconstrictor response and aggregation of platelets causing physical obstruction of capillaries, all of which, in turn may lead to reduced blood flow, aggravated ischemic damage and serious destruction in the normal tissue (6).

Neopterin is a marker of cellular immune response. Its levels are elevated in several conditions including autoimmune diseases such as systemic lupus erythematosus and rheumatoid arthritis; infections such as hepatitis and human immunodeficiency virus; cancers like hepatocellular, gastric and urothelial carcinomas; congestive heart failure, coronary artery disease , renal failure, transplant rejection and diabetes (7).

Neutrophil-to-lymphocyte ratio (NLR) was introduced as a potential marker to determine inflammation in renal and cardiac disorders (8).

Interleukin-10 is required for regulating immune functions by promoting the wide spread suppression of immune responses. IL-10 is effective at decreasing levels of proinflammatory cytokines that minimizes leukocyte maturation, recruitment and inflammation (9).

\section{SUBJECTS AND METHODS}

Study design: Cohort study gained the approval of Zagazig University Institutional Review Board before starting the study. A written informed consent was taken from all participants. It was carried out at Cardiology, Radiology, Internal Medicine and Clinical Pathology departments, Zagazig University Hospitals.

\section{SUBJECTS}

This study was applied on 2 groups of patients, one underwent coronary angiography for diagnostic and therapeutic purposes (45 cases) and the other underwent renal angiography (15 cases) using IV (high-osmolar) contrast media in Zagazig university hospitals.

The patients were classified after IV contrast adminstration into 2 groups :

- CIN group : The creatinine level was elevated either by $25 \%$ or more of the basal level or by $\geq 0.5 \mathrm{mg} / \mathrm{dl}$ above the basal level after 48 hours.

- No CIN group : Rise of the serum creatinine level less than $0.5 \mathrm{mg} / \mathrm{dl}$ above the basal level after 48 hours.

Exclusion criteria : Diabetes mellitus, Pregnancy and lactation , Presence of known malignancy , Evidence of current infection , inflammation, renal impairment or autoimmune diseases, Nephrotoxic drugs intake, Patients undergoing dialysis, Patients with nephrectomy and Shocked patients or with neurological dysfunction. 
All subjects of the study were subjected to the following:

- Full history \& full clinical examination.

- Blood samples were withdrawn for routine investigations (complete urine analysis. Fasting \& post prandial plasma sugar. Complete blood picture, serum creatinine and calculation of glomerular filtration rate using MDRD equation (basal, 24 and 48 hours after contrast administration). Serum uric acid, ESR, CRP and Cardiac enzymes (basal and 48 hours after contrast administration). Liver function tests, P.T, PTT and lipid profile.

- Blood samples were withdrawn for special investigations (serum neopterin, IL-10 and calculating neutrophil to lymphocyte ratio (basal, 24 and 48 hours after contrast administration).

Aim: To study the interrelation and levels of neopterin, interleukin-10 and neutrophil to lymphocyte ratio in contrast induced nephropathy.

\section{STATISTICAL ANALYSIS}

Data were analyzed with SPSS version 20.0 (statistical package for the Social Science). Quantitative data were expressed as mean \pm standard deviation (SD) or standard error (SE). $\mathrm{SE}=\mathrm{SD} /$ square root of patients number which was used in case of big SD, data were analyzed by independent sample, paired t test and one way analysis of variance (ANOVA). While qualitative data were expressed as number and percentage and were analyzed by Chi square (X2) test. Correlation was done using Pearson correlation test. The receiver operating characteristic (ROC) curve and $95 \%$ confidence interval (CI) was performed to determine cutoff values for the studied biomarkers. Sensitivity, specificity, positive predictive value (PPV) and negative predictive value (NPV) were determined. P-value was considered significant if $<0.05$ and highly significant if $<0.001$.

\section{RESULTS}

Table (1) shows the demographic basal data of studied groups (I \&II) highly statistical significant differences were found between studied groups (I \& II) as regard age and sex but no statistical significant difference was found between studied groups (I \& II) as regard BMI.

Table (2) shows the specific biomarkers data of studied groups (I \&II) before contrast adminstration a statistical significant difference was found between studied groups (I \& II) as regard CIN (number of patients) as 11 subjects of coronary angiography group while only 3 of renal angiography developed CIN but no statistical significant differences were found between studied groups (I \& II) as regard neopterin, IL 10 , serum creatinine, eGFR and NLR.

Table (3) shows the demographic basal data of of patients developed CIN and those without CIN after contrast adminstration, no statistical significant differences were found between them as regard age, sex and BMI.

Table (4) shows the routine laboratory basal data of patients developed CIN and those without CIN after adminstration of IV (highosmolar) contrast, no statistical significant differences were found between studied groups as regard ALT, AST, HB, PT, PTT, FBS and PPS but highly statistical significant differences was found between studied groups as regard serum total billirubin.

Table (5) shows the hematological and inflammatory basal laboratory data of patients developed CIN and those without CIN after contrast adminstration, no statistical significant differences were found between studied groups as regard cardiac enzymes, WBC and platelet count but highly statistical significant differences were found between studied groups as regard UA, ESR and CRP.

Table (6) shows the specific biomarkers basal data of patients developed CIN and NO CIN after contrast adminstration, no statistical significant differences were found between studied groups as regard neopterin, IL10, serum creatinine, eGFR and NLR.

Table (7) shows comaprison between CIN and NO CIN groups regarding differntial 
leucocytic count values .There was a highly statisitical significiant difference in WBC, neutrophil \& lymphocyte count and NLR while no statisitical significiant difference in monocyte count was found between the studied groups at 24 and 48 hours after contrast.

Table (8) shows comaprison between CIN and NO CIN groups regarding the serum inflammatory markers values.There was a highly statisitical significiant difference in ESR , CRP and uric acid between the studied groups before and $48 \mathrm{~h}$ after contrast.

Table (9) shows comaprison between CIN and NO CIN groups regarding the serum studied biomarkers values.There was a highly statisitical significiant increase in the serum neopterin, IL 10 levels and NLR comparing the studied groups (CIN more than NO CIN) at $24 \mathrm{~h}$ and $48 \mathrm{~h}$ after contrast adminstration.There was a highly statisitical significiant difference in the serum creatinine between the studied groups only at $48 \mathrm{~h}$ after contrast adminstration.
Table (10) shows no statistical significant correlation between $24 \mathrm{~h}$ serum Neopterin , IL10 and NLR versus each of Age (Years) , Hb level (g/dl) and MDRD ( $\mathrm{ml} / \mathrm{min})$ whereas highly statisitical sginificant correlations were found versus basal serum creatinine $(\mathrm{mg} / \mathrm{dl})$, basal serum neopterin $(\mathrm{nmol} / \mathrm{l})$, basal serum IL-10 (pg/ml) , basal $\mathrm{N} / \mathrm{L}$ ratio and basal billirubin $(\mathrm{mg} / \mathrm{dl})$ in CIN group

Table (11) shows no statistical significant correlation between $24 \mathrm{~h}$ serum Neopterin, IL10 and NLR versus each of Age (Years), basal serum creatinine $(\mathrm{mg} / \mathrm{dl})$, basal serum neopterin $(\mathrm{nmol} / \mathrm{l})$, basal serum $\mathrm{IL}-10(\mathrm{pg} / \mathrm{ml})$, $\mathrm{Hb}$ level (g/dl), MDRD (ml/min), basal N/L ratio and basal billirubin $(\mathrm{mg} / \mathrm{dl})$ in No CIN group.

Table (12) Area under the ROC curve of studied markers as predictors of CIN in the studied groups.

Table (13) shows the validity of the studied markers as predictors of CIN.

Table (1) Demographic data of studied groups before contrast adminstration using independent t- test.

\begin{tabular}{|c|c|c|c|c|c|}
\hline & & $\begin{array}{c}\text { CRONARY } \\
\text { ANGIOGRAPHY } \\
\text { (Group I) } \\
(\mathrm{n}=45) \\
(\text { Mean } \pm \text { SD }) \\
\end{array}$ & $\begin{array}{c}\text { RENAL } \\
\text { ANGIOGRAPHY } \\
\text { (Group II) } \\
(n=15) \\
(\text { Mean } \pm \text { SD }) \\
\end{array}$ & t-test & $\mathbf{P}$ \\
\hline \multirow{2}{*}{ Sex } & Male & 29 & 7 & & \\
\hline & Female & 16 & 8 & 8.3 & $<0.05 \mathrm{~S}$ \\
\hline \multicolumn{2}{|c|}{ Age (Years) } & $53.9 \pm 3.6$ & $34.8 \pm 3.5$ & 21.6 & $<0.001 \mathrm{HS}$ \\
\hline \multicolumn{2}{|c|}{$\begin{array}{c}\text { BMI }\left(\mathrm{Kg} / \mathrm{m}^{2}\right) \\
(18.5-24.9)\end{array}$} & $25.3 \pm 1.9$ & $25.5 \pm 1.5$ & 0.49 & $>0.05 \mathrm{NS}$ \\
\hline
\end{tabular}


Table (2) Specific biomarkers data of studied groups before contrast adminstration using independent $\mathrm{t}$ - test.

\begin{tabular}{|c|c|c|c|c|}
\hline & $\begin{array}{c}\text { CRONARY } \\
\text { ANGIOGRAPHY } \\
\text { (Group I) } \\
(n=45) \\
(\text { Mean } \pm \text { SD) } \\
\end{array}$ & $\begin{array}{c}\text { RENAL } \\
\text { ANGIOGRAPHY } \\
\text { (Group II) } \\
(n=15) \\
(\text { Mean } \pm \text { SD }) \\
\end{array}$ & t-test & $\mathbf{P}$ \\
\hline Neopterin $(<10 \mathrm{nmol} / \mathrm{l})$ & $8.8 \pm 2$ & $8.9 \pm 0.5$ & 0.7 & $>0.05 \mathrm{NS}$ \\
\hline IL $10(<18 \mathrm{pg} / \mathrm{ml})$ & $12.7 \pm 2.5$ & $13.2 \pm 3$ & -0.6 & $>0.05 \mathrm{NS}$ \\
\hline S.Cr $(0.7-1.2 \mathrm{mg} / \mathrm{dL})$ & $0.86 \pm 0.3$ & $0.9 \pm 0.2$ & 0.6 & $>0.05 \mathrm{NS}$ \\
\hline eGFR $(90-120 \mathrm{ml} / \mathrm{min} / 1.73 \mathrm{~m} 2)$ & $113.2 \pm 2.8$ & $115.8 \pm 3.2$ & -3.1 & $>0.05 \mathrm{NS}$ \\
\hline NLR (2-2.5) & $1.9 \pm 0.2$ & $1.9 \pm 0.1$ & -0.5 & $>0.05 \mathrm{NS}$ \\
\hline CIN (number of patients) & 11 & 3 & 4.3 & $<0.05 \mathrm{~S}$ \\
\hline Amount of contrast $(\mathrm{ml})$ & $181.4 \pm 33.7$ & $60.2 \pm 19.6$ & 12.9 & $<0.001 \mathrm{HS}$ \\
\hline
\end{tabular}

Table (3) Demographic basal data of patients developed CIN and those without CIN after contrast adminstration using independent $t$ - test.

\begin{tabular}{|c|c|c|c|c|c|}
\hline & & $\begin{array}{c}\text { CIN group } \\
(\mathrm{n}=14) \\
(\mathrm{Mean} \pm \mathrm{SD})(\end{array}$ & $\begin{array}{c}\text { No CIN } \\
(\mathrm{n}=46) \\
(\mathrm{Mean} \pm \mathrm{SD})\end{array}$ & t-test & $\mathbf{P}$ \\
\hline \multirow{2}{*}{ Sex } & Male & 9 & 27 & & \\
\hline & Female & 5 & 19 & 3 & $>0.05 \mathrm{NS}$ \\
\hline Age (Years) & & $56.1 \pm 6.9$ & $53.6 \pm 7.8$ & 1.1 & $>0.05 \mathrm{NS}$ \\
\hline $\begin{array}{c}\text { BMI }(\mathrm{Kg} / \mathrm{m} 2) \\
(18.5-24.9)\end{array}$ & & $25.3 \pm 2.1$ & $25.5 \pm 1.4$ & 0.47 & $>0.05 \mathrm{NS}$ \\
\hline
\end{tabular}

Table (4) Routine laboratory basal data of of patients developed CIN and those without CIN after contrast adminstration using independent $t$ - test.

\begin{tabular}{|c|c|c|c|c|}
\hline & $\begin{array}{c}\text { CIN group } \\
(\mathrm{n}=14) \\
(\mathrm{Mean} \pm \mathrm{SD})\end{array}$ & $\begin{array}{c}\text { No CIN } \\
(n=46) \\
(M e a n \pm S D)\end{array}$ & t-test & $\mathbf{P}$ \\
\hline $\operatorname{ALT}(10-40$ U/L) & $28.3 \pm 6.2$ & $31.8 \pm 6.8$ & 0.85 & $>0.05 \mathrm{NS}$ \\
\hline AST $(10-40 \mathrm{U} / \mathrm{L})$ & $23.9 \pm 4$ & $24.7 \pm 3.3$ & -0.8 & $>0.05 \mathrm{NS}$ \\
\hline HB (12.5-17 g/dl) & $12.9 \pm 1$ & $13.4 \pm 0.9$ & -1.9 & $>0.05 \mathrm{NS}$ \\
\hline Bil (Up to $1.2 \mathrm{mg} / \mathrm{dl})$ & $0.4 \pm 0.1$ & $1 \pm 0.1$ & -14.2 & $<0.001 \mathrm{HS}$ \\
\hline Alb $(3.5-4.9 \mathrm{~g} / \mathrm{dl})$ & $3.7 \pm 0.1$ & $3.7 \pm 0.2$ & 0.5 & $>0.05 \mathrm{NS}$ \\
\hline PT (11-14 sec) & $13 \pm 1$ & $12.5 \pm 1$ & 1.5 & $>0.05 \mathrm{NS}$ \\
\hline PTT (26-42 sec) & $34.2 \pm 4.7$ & $32.2 \pm 5.2$ & 1.3 & $>0.05 \mathrm{NS}$ \\
\hline FBS $\quad(70-100 \mathrm{mg} / \mathrm{dL})$ & $90.3 \pm 6.6$ & $83.7 \pm 9.4$ & 1.8 & $>0.05 \mathrm{NS}$ \\
\hline PPS (Less than $140 \mathrm{mg} / \mathrm{dL}$ ) & $139.2 \pm 3.2$ & $129.8 \pm 5.2$ & 21.4 & $>0.05 \mathrm{NS}$ \\
\hline
\end{tabular}


Table (5) Hematological and inflammatory basal data of of patients developed CIN and those without CIN after contrast adminstration using independent $t$ - test.

\begin{tabular}{|c|c|c|c|}
\hline & $\begin{array}{l}\text { CIN group } \\
(n=14) \\
(\text { Mean } \pm \text { SD })\end{array}$ & $\begin{array}{c}\text { No CIN } \\
(n=46) \\
(\text { Mean } \pm \text { SD })\end{array}$ & t-test \\
\hline WBC count $\quad\left(4-11 \quad 10^{3} / \mu \mathrm{L}\right)$ & $10.889 \pm 0.798$ & $8.716 \pm 1.421$ & $1.2>0.05 \mathrm{NS}$ \\
\hline Neutrophil count $\left(2-71^{3} / \mu L\right)$ & $5.808 \pm 0.99$ & $5.245 \pm 0.947$ & $1.8>0.05 \mathrm{NS}$ \\
\hline Lymphocyte count $\left(1-310^{3} / \mu \mathrm{L}\right)$ & $2.960 \pm 0.311$ & $2.859 \pm 0.387$ & $1.5>0.05 \mathrm{NS}$ \\
\hline PLT $\quad\left(150-450 \quad 10^{3} / \mu \mathrm{L}\right)$ & $323.214 \pm 63.194$ & $300.673 \pm 68.584$ & $41.1>0.05 \mathrm{NS}$ \\
\hline CPK - MB Up to $6.22 \mathrm{ng} / \mathrm{ml}$ & $6.3 \pm 0.5$ & $5.8 \pm 1.6$ & $1.8>0.05 \mathrm{NS}$ \\
\hline Troponin Up to $100 \mathrm{pg} / \mathrm{ml}$ & $85.1 \pm 16.1$ & $78.8 \pm 20.7$ & $1.0>0.05 \mathrm{NS}$ \\
\hline UA (2-7 mg/dl) & $7.3 \pm 0.3$ & $5.1 \pm 1$ & $8.4<0.001 \mathrm{HS}$ \\
\hline $\operatorname{ESR}(2-7 \mathrm{~mm} / \mathrm{hr})$ & $9.1 \pm 1.1$ & $5.7 \pm 2.4$ & $5.1<0.001 \mathrm{HS}$ \\
\hline CRP (1-5 mg/l) & $7.4 \pm 1.1$ & $3.4 \pm 1.1$ & $12.3<0.001 \mathrm{HS}$ \\
\hline
\end{tabular}

Table (6) Specific biomarkers basal data of of patients developed CIN and those without CIN after contrast adminstration using independent $t$ - test.

\begin{tabular}{|c|c|c|c|c|}
\hline & $\begin{array}{c}\text { CIN group } \\
(\mathrm{n}=14) \\
(\mathrm{Mean} \pm \mathrm{SD})\end{array}$ & $\begin{array}{c}\text { No CIN } \\
(n=46) \\
(M e a n \pm \text { SD })\end{array}$ & t-test & $\mathbf{P}$ \\
\hline Neopterin(<10 nmol/l) & $7.9 \pm 1.8$ & $6.6 \pm 2$ & 2.4 & $>0.05 \mathrm{NS}$ \\
\hline IL $10(<18 \mathrm{pg} / \mathrm{ml})$ & $13.8 \pm 1.7$ & $11.9 \pm 2.1$ & 2.5 & $>0.05 \mathrm{NS}$ \\
\hline $\mathrm{S.Cr}(0.7-1.2 \mathrm{mg} / \mathrm{dL})$ & $1 \pm 0.1$ & $0.9 \pm 0.3$ & 1.9 & $>0.05 \mathrm{NS}$ \\
\hline eGFR $(90-120 \mathrm{ml} / \mathrm{min} / 1.73 \mathrm{~m} 2)$ & $112.9 \pm 1.5$ & $114.7 \pm 2.8$ & -1.9 & $>0.05 \mathrm{NS}$ \\
\hline NLR (2-2.5) & $2 \pm 0.1$ & $1.9 \pm 0.1$ & 1.3 & $>0.05 \mathrm{NS}$ \\
\hline
\end{tabular}

Table (7) Comaprison between CIN and No CIN groups regarding differntial leucocytic count values

\begin{tabular}{|c|c|c|c|c|}
\hline & CIN group & NO CIN group & T- test & $\overline{\mathbf{P}}$ \\
\hline \multicolumn{5}{|c|}{$24 \mathrm{hs}$} \\
\hline WBC $\left(4-111^{3} / \mu \mathrm{L}\right)$ & $11.789 \pm 0.798$ & $8.812 \pm 1.358$ & 24.7 & $<0.001 \mathrm{HS}$ \\
\hline Neutrophil $\left(2-7 \quad 10^{3} / \mu \mathrm{L}\right)$ & $6.249 \pm 0.395$ & $5.255 \pm 0.829$ & 23.6 & $<0.001 \mathrm{HS}$ \\
\hline Lymphocyte $\left(1-31^{3} / \mu \mathrm{L}\right)$ & $2.138 \pm 0.508$ & $2.638 \pm 0.508$ & 12.6 & $<0.001 \mathrm{HS}$ \\
\hline Monocyte $\left(0.2-10^{3} / \mu \mathrm{L}\right)$ & $0.620 \pm 0.214$ & $0.615 \pm 0.123$ & 2.9 & $>0.05 \mathrm{NS}$ \\
\hline NLR (2-2.5) & $2.8 \pm 0.1$ & $1.9 \pm 0.1$ & 24.5 & $<0.001 \mathrm{HS}$ \\
\hline \multicolumn{5}{|c|}{$48 \mathrm{hs}$} \\
\hline WBC $\left(4-111^{3} / \mu \mathrm{L}\right)$ & $12.360 \pm 0.738$ & $8.916 \pm 1.421$ & 42.1 & $<0.001 \mathrm{HS}$ \\
\hline Neutrophil $\left(2-7 \quad 10^{3} / \mu \mathrm{L}\right)$ & $8.467 \pm 1.226$ & $5.232 \pm 0.685$ & 26.3 & $<0.001 \mathrm{HS}$ \\
\hline Lymphocyte $\left(1-31^{3} / \mu \mathrm{L}\right)$ & $1.907 \pm 0.499$ & $2.722 \pm 0.402$ & 27.3 & $<0.001 \mathrm{HS}$ \\
\hline Monocyte $\left(0.2-11^{3} / \mu \mathrm{L}\right)$ & $0.632 \pm 0.191$ & $0.635 \pm 0.143$ & 2.8 & $>0.05 \mathrm{NS}$ \\
\hline NLR (2-2.5) & $4.1 \pm 0.6$ & $1.9 \pm 0.2$ & 21.2 & $<0.001 \mathrm{HS}$ \\
\hline
\end{tabular}


Table (8) Comaprison between CIN and No CIN groups regarding the inflamatory markers serum values

\begin{tabular}{|c|c|c|c|c|}
\hline & CIN group & NO CIN group & T- test & $\overline{\mathbf{P}}$ \\
\hline \multicolumn{5}{|c|}{ Basal } \\
\hline ESR (2-7 mm/hr) & $9.1 \pm 1.1$ & $5.7 \pm 2.4$ & 5.1 & $<0.001 \mathrm{HS}$ \\
\hline CRP (1-5 mg/l) & $7.4 \pm 1.1$ & $3.4 \pm 1.1$ & 12.3 & $<0.001 \mathrm{HS}$ \\
\hline Uric acid $\quad(2-7 \mathrm{mg} / \mathrm{dl})$ & $7.3 \pm 0.3$ & $5.1 \pm 1$ & 8.4 & $<0.001 \mathrm{HS}$ \\
\hline \multicolumn{5}{|c|}{$48 \mathrm{hs}$} \\
\hline ESR (2-7 mm/hr) & $16.2 \pm 2$ & $6.4 \pm 1.4$ & 20.9 & $<0.001 \mathrm{HS}$ \\
\hline CRP (1-5 mg/l) & $13.6 \pm 1.5$ & $3.8 \pm 0.8$ & 31.3 & $<0.001 \mathrm{HS}$ \\
\hline Uric acid $(2-7 \mathrm{mg} / \mathrm{dl})$ & $7.6 \pm 0.3$ & $5.6 \pm 0.8$ & 9.7 & $<0.001 \mathrm{HS}$ \\
\hline
\end{tabular}

Table (9) Comaprison between CIN and No CIN groups regarding the studied biomarkers serum values

\begin{tabular}{|c|c|c|c|c|}
\hline & CIN group & NO CIN group & T- test & $\overline{\mathbf{P}}$ \\
\hline \multicolumn{5}{|c|}{ 24hs } \\
\hline Serum Neopterin $(<10 \mathrm{nmol} / \mathrm{l})$ & $21.6 \pm 2.7$ & $5.2 \pm 2$ & 24.7 & $<0.001 \mathrm{HS}$ \\
\hline Serum IL10 (<18 pg/ml) & $27.1 \pm 1.4$ & $13.4 \pm 2$ & 23.6 & $<0.001 \mathrm{HS}$ \\
\hline Serum Creatinine (0.7-1.2 mg/dL) & $1.1 \pm 0.1$ & $0.9 \pm 0.2$ & 2.6 & $>0.05 \mathrm{NS}$ \\
\hline NLR (2-2.5) & $2.8 \pm 0.1$ & $1.9 \pm 0.1$ & 24.5 & $<0.001 \mathrm{HS}$ \\
\hline \multicolumn{5}{|c|}{ 48hs } \\
\hline Serum Neopterin $(<10 \mathrm{nmol} / \mathrm{l})$ & $28.1 \pm 2.1$ & $6 \pm 1.6$ & 42.1 & $<0.001 \mathrm{HS}$ \\
\hline Serum IL10 $(<18$ pg/ml $)$ & $34.1 \pm 2.8$ & $14.5 \pm 2.3$ & 26.3 & $<0.001 \mathrm{HS}$ \\
\hline Serum Creatinine (0.7-1.2mg/dL) & $2 \pm 0.1$ & $0.6 \pm 0.2$ & 27.3 & $<0.001 \mathrm{HS}$ \\
\hline NLR (2-2.5) & $4.1 \pm 0.6$ & $1.9 \pm 0.2$ & 21.2 & $<0.001 \mathrm{HS}$ \\
\hline
\end{tabular}

Table (10) Correlation coefficient (r) value of serum Neopterin (nmol/l), serum IL 10 (pg/ml) and NLR after 24 hours versus some studied parameters in CIN group

\begin{tabular}{|c|c|c|c|c|c|c|}
\hline \multirow{2}{*}{$\begin{array}{l}\text { CIN Group } \\
24 \text { hrs }\end{array}$} & \multicolumn{2}{|c|}{$\begin{array}{c}\text { Neopterin } \\
(\text { nmol/l) }\end{array}$} & \multicolumn{2}{|c|}{$\begin{array}{c}\text { IL } 10 \\
(p g / m l)\end{array}$} & \multicolumn{2}{|c|}{$N L R$} \\
\hline & $\mathbf{R}$ & $\mathbf{P}$ & $\mathbf{R}$ & $\mathbf{P}$ & $\mathbf{R}$ & $\mathbf{P}$ \\
\hline Age (yrs) & 0.33 & $>0.05 \mathrm{NS}$ & 0.31 & $>0.05 \mathrm{NS}$ & 0.29 & $>0.05 \mathrm{NS}$ \\
\hline $\begin{array}{c}\text { Basal serum creatinine } \\
(0.7-1.2 \mathrm{mg} / \mathrm{dL})\end{array}$ & 0.69 & $<0.001 \mathrm{HS}$ & 0.67 & $<0.001 \mathrm{HS}$ & 0.65 & $<0.001 \mathrm{HS}$ \\
\hline $\begin{array}{c}\text { Basal serum Neopterin } \\
(<10 \mathrm{nmol} / \mathrm{l})\end{array}$ & 0.49 & $<0.001 \mathrm{HS}$ & 0.51 & $<0.001 \mathrm{HS}$ & 0.47 & $<0.001 \mathrm{HS}$ \\
\hline $\begin{array}{c}\text { Basal serum IL } 10 \\
(<18 \mathrm{pg} / \mathrm{ml})\end{array}$ & 0.47 & $<0.001 \mathrm{HS}$ & 0.52 & $<0.001 \mathrm{HS}$ & 0.51 & $<0.001 \mathrm{HS}$ \\
\hline$H b(g / d l)$ & 0.149 & $>0.05 \mathrm{NS}$ & 0.14 & $>0.05 \mathrm{NS}$ & 0.19 & $>0.05 \mathrm{NS}$ \\
\hline$M D R D(\mathrm{ml} / \mathrm{min})$ & -0.24 & $>0.05 \mathrm{NS}$ & -0.22 & $>0.05 \mathrm{NS}$ & -0.21 & $>0.05 \mathrm{NS}$ \\
\hline Basal NLR (2-2.5) & 0.48 & $<0.001 \mathrm{HS}$ & 0.58 & $<0.001 \mathrm{HS}$ & 0.53 & $<0.001 \mathrm{HS}$ \\
\hline $\begin{array}{l}\text { Basal Billirubin } \\
\text { (Up to } 1.2 \mathrm{mg} / \mathrm{dl})\end{array}$ & -0.51 & $<0.001 \mathrm{HS}$ & -0.61 & $<0.001 \mathrm{HS}$ & -0.58 & $<0.001 \mathrm{HS}$ \\
\hline
\end{tabular}


Table (11) Correlation coefficient (r) value of serum Neopterin ( nmol/l), serum IL 10 (pg/ml) and NLR after 24 hours versus some studied parameters in NO CIN group

\begin{tabular}{|c|c|c|c|c|c|c|}
\hline \multirow{2}{*}{$\begin{array}{c}\text { NO CIN Group } \\
24 \text { hrs }\end{array}$} & \multicolumn{2}{|c|}{$\begin{array}{c}\text { Neopterin } \\
(\mathrm{nmol} / \mathrm{l})\end{array}$} & \multicolumn{2}{|c|}{$\begin{array}{c}\text { IL } 10 \\
(p g / m l)\end{array}$} & \multicolumn{2}{|c|}{$N L R$} \\
\hline & $\mathbf{R}$ & $\mathbf{P}$ & $\mathbf{R}$ & $\mathbf{P}$ & $\mathbf{R}$ & $\mathbf{P}$ \\
\hline Age (yrs) & 0.4 & $>0.05 \mathrm{NS}$ & 0.22 & $>0.05 \mathrm{NS}$ & 0.27 & $>0.05 \mathrm{NS}$ \\
\hline $\begin{array}{c}\text { Basal serum creatinine } \\
(0.7-1.2 \mathrm{mg} / \mathrm{dL})\end{array}$ & 0.24 & $>0.05 \mathrm{NS}$ & 0.15 & $>0.05 \mathrm{NS}$ & 0.16 & $>0.05 \mathrm{NS}$ \\
\hline $\begin{array}{c}\text { Basal serum Neopterin } \\
(<10 \mathrm{nmol} / \mathrm{l})\end{array}$ & 0.23 & $>0.05 \mathrm{NS}$ & 0.27 & $>0.05 \mathrm{NS}$ & 0.18 & $>0.05 \mathrm{NS}$ \\
\hline $\begin{array}{c}\text { Basal serum IL } 10 \\
(<18 \mathrm{pg} / \mathrm{ml})\end{array}$ & 0.29 & $>0.05 \mathrm{NS}$ & 0.18 & $>0.05 \mathrm{NS}$ & 0.22 & $>0.05 \mathrm{NS}$ \\
\hline$H b(g / d l)$ & 0.073 & $>0.05 \mathrm{NS}$ & -0.149 & $>0.05 \mathrm{NS}$ & 0.073 & $>0.05 \mathrm{NS}$ \\
\hline$M D R D(\mathrm{ml} / \mathrm{min})$ & -0.21 & $>0.05 \mathrm{NS}$ & -0.23 & $>0.05 \mathrm{NS}$ & -0.29 & $>0.05 \mathrm{NS}$ \\
\hline Basal NLR (2-2.5) & 0.145 & $>0.05 \mathrm{NS}$ & 0.29 & $>0.05 \mathrm{NS}$ & 0.45 & $>0.05 \mathrm{NS}$ \\
\hline $\begin{array}{l}\text { Basal Billirubin } \\
\text { (Up to } 1.2 \mathrm{mg} / \mathrm{dl})\end{array}$ & 0.3 & $>0.05 \mathrm{NS}$ & 0.19 & $>0.05 \mathrm{NS}$ & 0.15 & $>0.05 \mathrm{NS}$ \\
\hline
\end{tabular}

Table (12) Area under the ROC curve of studied markers as Predictors of CIN in the studied groups

\begin{tabular}{|c|c|c|c|c|}
\hline Predictor & $\begin{array}{c}\text { Area under } t \\
\text { he ROC curv } \\
\text { e } \\
(A U C)\end{array}$ & $\begin{array}{l}\text { Standard } \\
\text { Error }^{a}\end{array}$ & $\begin{array}{c}\text { 95\% Confidence } \\
\text { interval }^{b}\end{array}$ & $\begin{array}{c}\text { Significance level P } \\
(\text { Area }=0.5)\end{array}$ \\
\hline$N L R$ & 0.757 & 0.0661 & 0.629 to 0.858 & 0.0001 \\
\hline $\boldsymbol{U A}$ & 0.997 & 0.00379 & 0.934 to 1.000 & $<0.0001$ \\
\hline$C R P$ & 0.901 & 0.066 & 0.940 to 1.000 & $<0.0001$ \\
\hline Bil & 0.965 & 0.204 & 0.940 to 1.001 & $<0.0001$ \\
\hline$E S R$ & 0.87 & 0.0451 & 0.758 to 0.943 & $<0.0001$ \\
\hline $\begin{array}{c}\text { Neopteri } \\
n\end{array}$ & 0.884 & 0.0531 & 0.775 to 0.952 & $<0.0001$ \\
\hline ILI0 & 0.911 & 0.0408 & 0.809 to 0.969 & $<0.0001$ \\
\hline
\end{tabular}


Table (13) Cut-off value of studied markers as Predictors of CIN in the studied groups

\begin{tabular}{|c|c|c|c|c|c|c|c|c|c|}
\hline $\begin{array}{c}\text { Predicted } \\
\text { outcome }\end{array}$ & $\begin{array}{c}\text { Cut- } \\
\text { off }\end{array}$ & $\begin{array}{c}\text { Sensitivity } \\
\% \\
\end{array}$ & $95 \% C I$ & $\begin{array}{c}\text { Specificity } \\
\%\end{array}$ & $95 \% C I$ & $\begin{array}{c}P P V \\
\% \\
\end{array}$ & $95 \% C I$ & $\begin{array}{c}N P V \\
\% \\
\end{array}$ & $95 \% C I$ \\
\hline NLR (24) & $>2.9$ & 100 & $\begin{array}{l}76.8- \\
100.0\end{array}$ & 36.96 & $\begin{array}{c}23.2- \\
52.5\end{array}$ & 32.6 & $\begin{array}{c}19.1- \\
48.5\end{array}$ & 100 & 80.5100 .0 \\
\hline$U A(0)$ & $>7.6$ & 100 & $\begin{array}{l}76.8- \\
100.0\end{array}$ & 95.65 & $\begin{array}{c}85.2- \\
99.5\end{array}$ & 87.5 & $\begin{array}{r}61.7- \\
98.4\end{array}$ & 100 & 92.0100 .0 \\
\hline$C R P(0)$ & $>7$ & 92.68 & $\begin{array}{c}66.1- \\
99.8\end{array}$ & 95.65 & $\begin{array}{r}92.3- \\
99.8\end{array}$ & 86.7 & $\begin{array}{r}76.8- \\
98.1\end{array}$ & 97.8 & $92.3-99.1$ \\
\hline Bil (0) & $\leq 0.5$ & 92.86 & $\begin{array}{c}76.8- \\
98.2\end{array}$ & 93.48 & $\begin{array}{c}92.3- \\
99.4\end{array}$ & 81.3 & $\begin{array}{r}70.8- \\
95.8\end{array}$ & 96.1 & $90.3-98.4$ \\
\hline $\operatorname{ESR}(0)$ & $>9$ & 92.86 & $\begin{array}{c}66.1- \\
99.8\end{array}$ & 78.26 & $\begin{array}{c}63.6- \\
89.1\end{array}$ & 56.5 & $\begin{array}{c}34.5- \\
76.8\end{array}$ & 97.3 & $85.8-99.9$ \\
\hline $\begin{array}{c}\text { Neopterin } \\
\text { (24) }\end{array}$ & $>15.4$ & 85.71 & $\begin{array}{r}57.2- \\
98.2\end{array}$ & 84.78 & $\begin{array}{c}71.1- \\
93.7\end{array}$ & 63.2 & $\begin{array}{c}38.4- \\
83.7\end{array}$ & 95.1 & $83.5-99.4$ \\
\hline ILI0 (24) & $>23.6$ & 85.71 & $\begin{array}{r}57.2- \\
98.2\end{array}$ & 84.78 & $\begin{array}{c}71.1- \\
93.7\end{array}$ & 63.2 & $\begin{array}{c}38.4- \\
83.7\end{array}$ & 95.1 & $83.5-99.4$ \\
\hline
\end{tabular}

\section{DISCUSSION}

Acute kidney injury (AKI), previously referred to as acute renal failure (ARF), represents a significant and devastating problem in clinical medicine. The incidence of AKI varies from $5 \%$ of hospitalized patients to $30-50 \%$ of patients in intensive care units .Often AKI manifests as a transient rise in serum creatinine and is managed conservatively; however, a group of patients, often with significant comorbidity, require temporary renal replacement therapy (1).

Contrast medium-induced damage is the third highest cause of hospital-acquired acute renal failure, and in half of these cases, it occurs after invasive cardiac procedures. It is usually defined as impairment of renal function occurring within $48 \mathrm{~h}$ after administration of contrast media and manifested by an absolute increase in serum creatinine concentration of at least $0.5 \mathrm{mg} / \mathrm{dl}$ or by a relative increase of at least $25 \%$ from the baseline value (3).

Acute renal injury is typically diagnosed by measuring serum creatinine. Unfortunately, creatinine is an unreliable indicator during acute changes in kidney function . It is affected by extra renal factors that include an individual mass and extracellular volume irrespective of the level of glomerular filtration rate; it does not increase immediately post kidney injury and occasionally exhibits a downward trend post kidney injury. Additionally, a significant loss of renal injury is accompanied by normal serum creatinine levels. Thus, there is a need for rapidly available, sensitive and specific biomarkers for AKI that would allow early prediction at a time when intensive care optimization can be performed (10).

Therefore, the aim of this study to clarify the interrelation and levels of neopterin, interleukin-10 and neutrophil to lymphocyte ratio in patients with normal serum creatinine undergoing percutaneous coronary angiography and renal angiography using IV (high-osmolar) contrast media.

There were statsitcal significant differences in the age and sex of patients between the two groups which can be explained by the increased incidence of cronary artery disease in male sex and in older age group, as proposed by Debra et al., (1986) (11). This was also associated with satisitical significant difference in the lipid profile between the 2 groups, while there was 
no statisitical significant difference in the other laboratory parameters.

There was a statsitical significant difference between the 2 groups in the amount of contrast media (about 150-200 $\mathrm{ml}$ in coronary angiography group versus $50-70 \mathrm{ml}$ in renal angiography group due to the nature of both proceduers which was associated with increased number of CIN patients in the coronary anigiography group which goes in agreement with Diaz-Sandoval et al., (2002) (12).

The reported incidence of contrast-induced nephropathy varies widely, ranging from $0 \%$ to $50 \%$ (13) . This variability results from differences in the presence or absence of risk factors, definition of contrast-induced nephropathy, the contrast agent characteristics including the type, amount, duration and route of administration and length of follow-up.

There are several risk factors that may influence the development of CIN, such as dehydration, contrast media volume, diabetes mellitus, hypertension and old age. In patients without risk factors, the incidence may be as low as $2 \%$. In those with risk factors, such as diabetes, the rate rises to $9 \%$, and to as high as $90 \%$ in patients with diabetic nephropathy. Therefore, the number and the type of preexisting risk factors directly influence the incidence of renal insufficiency (14).

About $25 \%$ of the present studied patients (14 patients) fulfilled the criteria of contrast nephropathy with more cases in the percuteanous coronary angiography group (11 patients) as among all procedures utilizing contrast media for diagnostic or therapeutic purposes, coronary angiography and percutaneous coronary interventions (PCI) are associated with the highest rates of CIN mainly related not only to the intra-arterial injection and to the high dosage of the contrast necessary but also to the type of patients (advanced age, one or more comorbid conditions and more advanced vascular diseases such as hypertension).
In our study age was not associated with the risk of CIN which goes in agreement with Diogo et al., 2014 (15) who found a nonsignificant association between ages $>65$ years and risk for CIN . Conversely Marenzi et al., 2014 (16) in an observational study of patients undergoing PCI, reported that age exceeding 75 years predicted a 5-fold risk for developing CIN (OR 5.28, 95\% 1.98-14.05, p<0.001) as explained by decreasing glomerular filtration rate following structural changes due to fibrotic changes to the kidneys with advancing age, calcification and likely increased presence of comorbidities in elderly patients.

Proposed pathophysiologic mechanisms through which contrast administration may potentiate renal injury include oxidative stress, free radical damage and endothelial dysfunction, renal vasoconstriction resulting in medullary hypoxia and direct cytotoxic effects of the contrast agents (17).

Experimental studies have shown a significant relationship between inflammation and acute renal injury. First, renal vascular endothelium and tubular epithelium undergo structural and functional changes due to ischemia, sepsis or nephrotoxic effects. At the next stage, inflammatory cells particularly neutrophils infiltrate damaged tissue leading to further renal destruction. Overactivation of neutrophils increases the release of cytokines, reactive oxygen products and many enzymes, which in turn, enhances vascular permeability and disrupts endothelial functions, all of which, in turn may lead to reduced blood flow, aggravated ischemic damage and serious destruction in the normal tissue (18).

Neopterin, a pyrazino-pyrimidine compound, is synthesized by monocytes and macrophages in response to interferon $\gamma$ produced by activated T cells. Neopterin levels are elevated in conditions of $\mathrm{T}$-cell or macrophages activation. Increased neopterin levels are observed in diseases with intensified monocyte/macrophage activity. Neopterin measurements in body fluids provide 
informations on a current state of cellular immune response and frequently help prediction of disease progression. The release of neopterin begins 3 days before $\mathrm{T}$ cells proliferation reaches maximum and an increase in neopterin production can be observed about one week before the appearance of specific antibodies .Therefore, neopterin may be of clinical use as an early inflammation marker (19).

In our study, Neopterin levels were found to be higher in CIN group at 24 and 48 hours than in NO CIN group .

In recent studies, evidence strongly implicates inflammation in the mechanism of CIN and other forms of ischaemic AKI. Cytokine induced inflammation plays a significant role as predictive biomarkers of disease and prognosis. Increased levels of cytokines are associated with adverse outcomes in patients with AKI. CIN is characterised by an imbalance of inflammatory cytokines namely TNF $\alpha$ and IL10. TNF $\alpha$ as a proinflammatory cytokine accelerates CIN and therefore poorer clinical outcomes, while IL10 accelerates recovery $\mathbf{( 2 0 )}$.

Interleukin-10 is required for regulating immune functions by promoting the wide spread suppression of immune responses. IL-10 is effective at decreasing levels of proinflammatory cytokines that minimizes leukocyte maturation, recruitment and inflammation and therefore protects the kidney following insult and hastens renal recovery (9).

In our study, IL 10 levels were found to be higher in CIN group at 24 and 48 hours than in NO CIN group while no difference in baseline values in contrary to Chang et al., 2013 (20) who reported reduced baseline IL10 levels in CIN patients compared to controls $(1.02 \pm 1.14$ vs. $2.78 \pm 4.73 \mathrm{pg} / \mathrm{ml}, \mathrm{p}=0.008)$ and Justor Banda et al., 2016 (21) who did not find any significance between IL10 level and CIN.

White blood cell count and subtypes are recognized as inflammatory markers in cardiovascular diseases. NLR is calculated as the proportion of 2 inflammatory markers (neutrophil and lymphocyte) and therefore has a strong predictive value. NLR is a simple parameter to assess easily the inflammatory status of a subject. It has proven its usefulness in the stratification of mortality in major cardiac events, as a strong prognostic factor in several types of cancers, or as a predictor and a marker of inflammatory or infectious pathologies and postoperative complications ( 8).

In our study, NLR levels were found to be higher in CIN group at 24 and 48 hours than in NO CIN group and showed a significant and independent relationship with the development of contrast induced nephropathy, which appears to be supportive of the abovementioned view which goes in agreement with Ahmet Kaya et al., 2014 (22) and Alparslan Kurtul et al., 2016 (23).

CRP is a widely used biochemical marker for acute inflammation. It rises above normal limits within 2 hours of onset of inflammation, peaks in 48 hours and has a steady half-life that causes a rapid and predictable fall once the inflammation has stopped. Possible explanations of association of CRP with CIN include: Firstly, increased CRP is associated with platelet and clotting system activation, which may decrease renal blood flow. Secondly, CRP can mediate enhanced expression of adhesion molecules, a potent chemo attractant, and plasminogen activator inhibitor-1 and a reduction in nitric oxide production, resulting in endothelium dysfunction and altering the vascular equilibrium toward a proinflammatory, prothrombotic and vasoconstrictive state (24).

In our study; we found a significant relationship between increased pre-procedural and post-procedural CRP levels and development of contrast nephropathy, which appears to be supportive of the abovementioned view which goes in agreement with Lazaros et al., 2016 (24) and Ibrahim Rencuzogullari et al., 2018 (25). 
In our study; we found a significant relationship between increased pre-procedural and post-procedural ESR levels and development of CIN, which appears to be supportive of the above-mentioned view which goes in agreement with Ying Yuan et al., 2017 (26).

Enhanced reactive oxygen species (ROS) generation in clinical conditions predisposing to CIN and the evidence for enhanced ROS formation following exposure to contrast media, highlight the possibility that oxidative stress may play an important role in the pathogenesis of CIN. Antioxidant agents such as ascorbic acid and acetylcysteine showed promising results for the prevention of CIN (27).

Uric acid is the end product of purine metabolism in humans. Elevated serum uric acid levels are related to various pathologic processes such as endothelial dysfunction, inflammation, activation of the reninangiotensin system, inhibition of the nitric oxide system and increased oxidative stress . It is observed that after radioconstrast exposure, excretion of uric acid in the urine increases and may predispose to crystallization, especially in some conditions as in acidic urine occurring during volume depletion.These urate crystals are proinflammatory and may induce tubular injury (28).

In our study; we found a significant relationship between increased pre-procedural and post-procedural UA levels and development of contrast nephropathy, which appears to be supportive of the abovementioned view which goes in agreement with

Zuo et al., 2016 (29) and Mendi et al., 2017 (28). Conversely, some studies did not find any association between serum UA and CIN Karabulut et al., 2014 (30).

Bilirubin, once considered simply the metabolic end-product of heme degradation, has now emerged as an important endogenous anti-inflammatory and antioxidant molecule. Accumulating evidence suggests that bilirubin may be part of a cell defense strategy in response to oxidative stress. Previous studies indicated that bilirubin serves as a physiological antioxidant in ischemia-reperfusion and may prevent experimental atherosclerosis (31).

In our study, we found a significant relationship between decreased basal serum total billirubin level and development of CIN , which suggest that enhanced oxidative stress and insufficient anti-oxidative defense mechanism are the potential pathophysiology in patients developing CIN which goes in agreement with Huang et al., 2012 (32) and Vuruşkan et al., 2017 (33).

There were statisitical sginificant correlations between $24 \mathrm{~h} \& 48 \mathrm{~h}$ serum neopterin ( $\mathrm{nmol} / \mathrm{l})$, interleukin $10(\mathrm{pg} / \mathrm{ml})$, neutrophil to lymphocyte ratio and each of the following : basal serum creatinine $(\mathrm{mg} / \mathrm{dl})$, basal serum neopterin (nmol/l) , basal serum interleukin $10(\mathrm{pg} / \mathrm{ml})$, basal neutrophil to lymphocyte ratio, basal billirubin $(\mathrm{mg} / \mathrm{dl})$ and amount of IV (high-osmolar) contrast $(\mathrm{ml})$ in CIN group, while there was no statistical significant correlations between $24 \mathrm{~h} \& 48 \mathrm{~h}$ serum neopterin $(\mathrm{nmol} / \mathrm{l})$, interleukin 10 $(\mathrm{pg} / \mathrm{ml})$, neutrophil to lymphocyte ratio and each of the following: basal serum creatinine $(\mathrm{mg} / \mathrm{dl})$, basal serum neopterin $(\mathrm{nmol} / \mathrm{l})$, basal serum interleukin $10(\mathrm{pg} / \mathrm{ml})$, basal neutrophil to lymphocyte ratio, basal billirubin $(\mathrm{mg} / \mathrm{dl})$ and amount of IV (high-osmolar) contrast (ml) in No CIN group.

In our study; we found that the sensitivity and specificity of serum neopterin and IL 10 at $24 \mathrm{hrs}$ after contrast adminstration was $85.71 \%$ $\& 84.78 \%, 85.71 \%$ \& $84.78 \%$ respectively.

In our study; we found that the sensitivity and specificity of NLR at 24hrs after contrast adminstration was $100 \%$ \& $36.96 \%$ while Ahmet Kaya et al., 2014 (22) found 75\% sensitivity \& $65 \%$ specificity and Alparslan Kurtul et al., 2016 (23) demonstrated 73\% specificity \& $70 \%$ sensitivity.

In our study; we found that the sensitivity and specificity of CRP before contrast 
adminstration was $92.68 \%$ \& $95.65 \%$ while Ying Yuan et al., 2017 (26) found $72.4 \%$ sensitivity \& $61.8 \%$ specificity.

In our study; we found that the sensitivity and specificity of ESR before contrast adminstration was $92.86 \%$ \& $78.26 \%$ while Ying Yuan et al., 2017 (26) found $46.6 \%$ sensitivity \& $76.4 \%$ specificity.

In our study; we found that the sensitivity and specificity of UA before contrast adminstration was $100 \% \& 95.65 \%$ while Mendi et al., 2017 (28) found $70 \%$ sensitivity $\& 67 \%$ specificity.

\section{CONCLUSION}

Serum neopterin, IL 10 and neutrophil to lymphocyte ratio proved that they can be used as early biomarkers of contast induced nephropathy instead of serum creatinine as they rise 24 hours before any change in the serum creatinine. Decreased serum total bilirubin levels had a higher incidence of CIN after the use of contrast media. Measuring ESR \& CRP levels at admission may offer additional assistance in predicting the development of CIN. Elevated serum uric acid level is independently associated with an increased risk of CIN. Future research is needed to determine whether urate-lowering therapy has beneficial effects for reducing the incidence of CIN. Application of treatments with antiinflammatory properties may be worthy for further investigation in the prevention of CIN or in the early intervention.

\section{REFERENCES}

(1) Devarajan P (2006): Update on Mechanisms of Ischemic Acute Kidney Injury. Journal of the American Society of Nephrology, 17 (6), pp:15031520.

(2) Briguori C, Tavano D \& Colombo A (2003): Contrast agent - associated nephrotoxicity. Prog Cardiovasc Dis; 45: pp:493-503.

(3) Kaya A, Kurt M, Tanboga IH et al. (2013): Relation of neutrophil to lymphocyte ratio with the presence and severity of stable coronary artery disease Clinical and Applied Thrombosis/Hemostasis, 20 (5), pp:473-477.
(4) Solomon R \& Dauerman HL (2010): Contrastinduced acute kidney injury. Circulation, 122 (23), pp: 2451-2455.

(5) Goldenberg I \& Matetzky S (2005): Nephropathy induced by contrast media: pathogenesis, risk factors and preventive strategies. Canadian Medical Association Journal, 172 (11), pp:1461-1471.

(6) Akcay A , Nguyen Q \& Edelstein CL ( 2009): Mediators of inflammation in acute kidney injury.Mediators Inflamm.13, pp:70-72.

(7) Lhee HY, Kim H, Kwan JJ et al. (2006): The clinical significance of serum and urinary neopterin levels in several renal diseases," Journal of Korean medical science, 21(4), pp:678-682.

(8) Turkmen K , Guney I , Yerlikaya FH et al. (2012): Relationship between neutrophil-to lymphocyte ratio and inflammation in end-stage renal disease patients. Renal failure, 34 (2), pp:155-159.

(9) Tang-Feldman YJ, Lochhead GR, Lochhead SR et al. (2011): Interleukin-10 repletion suppresses pro-inflammatory cytokines and decreases liver pathology without altering viral replication in murine cytomegalovirus -infected IL-10 knockout mice. Inflammation research, 60 (3), pp:233-243.

(10)Schley G, Köberle C, Manuilova E et al. (2015): Comparison of Plasma and Urine Biomarker Performance in Acute Kidney Injury. PloS one, 10 (12), p:e0145042.

(11)Debra J and William B (1986): Patterns of coronary heart disease morbidity and mortality in the sexes: A 26-year follow-up of the Framingham population, American heart journal, 111 (2), pp:383-390.

(12)Diaz-Sandoval LJ, Kosowsky BD and Losordo DW (2002): Acetylcysteine to prevent angiography-related renal tissue injury (the APART trial). The American journal of cardiology, 89 (3), pp:356-358.

(13) Gami AS and Garovic VD (2004): Contrast nephropathy after coronary angiography. Mayo Clin Proc.(79), pp:211-19.

(14) Abacı O, Harmankaya O, Kocas B et al. (2015): Long-term follow up of patients at high risk for nephropathy after contrast exposure. Angiology, 66 (6), pp:514-518.

(15)Diogo LP, Bahlis LF \& Carvalhal GF (2014): Computerized tomography contrast induced nephropathy (CIN) among adult inpatients. Jornal Brasileiro de Nefrologia, 36 (4), pp:446-450.

(16)Marenzi G, Lauri G, Assanelli E et al. (2014): Contrast induced nephropathy in patients 
undergoing primary angioplasty for acute myocardial infarction. Journal of the American College of Cardiology ;44 (9):1780-5.

(17) Mamoulakis C, Tsarouhas K, Fragkiadoulaki I et al. (2017): Contrast-induced nephropathy: Basic concepts, pathophysiological implications and prevention strategies. Pharmacology \& therapeutics. Dec;180: pp:99-112.

(18)Sato A, Aonuma K, Watanabe M et al. (2017): Association of contrast-induced nephropathy with risk of adverse clinical outcomes in patients with cardiac catheterization: From the CINC-J study. International journal of cardiology, 227, pp:424429.

(19) Emin Ozgur Akgul, İbrahim Aydın , Tuncer Caycs et al. (2013): The indicator of cellular immune response in body fluids: Neopterin Gulhane Med. J, 55, pp:237-243.

(20)Chang CF, Lu TM, Yang WC et al. (2013): Gene polymorphisms of interleukin-10 and tumor necrosis factor-alpha are associated with contrastinduced nephropathy. American journal of nephrology, 37 (2), pp:110-117.

(21) Justor B , Duarte R , Dickens C et al. (2016): Risk Factors and Outcomes for Contrast Induced Nephropathy among Hospitalized South Africans. SAMJ: South African Medical Journal, 106 (7), pp:699-703.

(22) Ahmet Kaya, Yasemin Kaya, Selim Topc u et al. (2014): Neutrophil-to-Lymphocyte Ratio Predicts Contrast-Induced Nephropathy in Patients Undergoing Primary Percutaneous Coronary Intervention Angiology, 65 (1), pp:51-56.

(23) Alparslan Kurtul, Mikail Yarlioglues, Mustafa Duran et al. (2016): Association of Neutrophil-tolymphocyte Ratio with Contrast-induced Nephropathy in Patients with Non-ST-elevation Acute Coronary SyndromeTreated with Percutaneous Coronary Intervention Heart, Lung and Circulation, 25 (7), pp:683-690.

(24)Lazaros G, Zografos T, Oikonomou E et al. (2016) : Usefulness of C-Reactive Protein as a Predictor of Contrast-Induced Nephropathy After Percutaneous Coronary Interventions in Patients With Acute Myocardial Infarction and Presentation of a New Risk Score (Athens CIN Score). American Journal of Cardiology, 118 (9), pp:13291333.

(25) Ibrahim Rencuzogullari, Metin Çağdaş \& Süleyman Karakoyun (2018): Association of Syntax Score II with Contrast-induced Nephropathy and Hemodialysis Requirement in Patients with ST Segment Elevation Myocardial
Infarction Undergoing Primary Percutaneous Coronary Intervention Korean circulation journal, 48 (1), pp:59-70.

(26) Yuan Y, Qiu H, Hu X et al. (2017): Predictive value of inflammatory factors on contrast induced acute kidney injury in patients who underwent an emergency percutaneous coronary intervention. Clinical cardiology, 40 (9), pp:719725.

(27)Park SH, Jeong MH, Park IH et al. (2016): Effects of combination therapy of statin and $\mathrm{N}$ acetylcysteine for the prevention of contrastinduced nephropathy in patients with ST-segment elevation myocardial infarction undergoing primary percutaneous coronary intervention. International journal of cardiology, 212, pp:100106.

(28)Mendi MA, Afsar B, Oksuz F et al. (2017): Uric Acid is a Useful Tool to Predict Contrast-Induced Nephropathy. Angiology. Aug; 68 (7): pp:627632.

(29)Zuo T, Jiang L, Mao S et al (2016): Hyperuricemia and contrast-induced acute kidney injury: A systematic review and meta-analysis. International journal of cardiology, 224, pp:286294.

(30) Karabulut A, Sahin I, Ilker Avci I et al. (2014): Impact of serum alkaline phosphatase level on the pathophysiologic mechanism of contrast-induced nephropathy. Kardiologia Polska (Polish Heart Journal), 72 (10), pp:977-982.

(31)Sticova E \& Jirsa M (2013): New insights in bilirubin metabolism and their clinical implications. World Journal of Gastroenterology: WJG, 19 (38), p:6398.

(32)Huang SS, Huang PH, Wu TC et al (2012): Association of Serum Bilirubin with ContrastInduced Nephropathy and Future Cardiovascular Events in Patients Undergoing Coronary Intervention. PLoS ONE 7 (8):p:e42594.

(33) Vuruşkan E \& Saraçoğlu E (2017): Bilirubin levels are associated with contrast-induced nephropathy in peripheral artery disease. Angiology, 68 (8), pp:728-733. 